\title{
Reduction in Acute Stroke Admissions during the COVID-19 Pandemic: Data from a National Stroke Registry
}

\author{
Carmit Libruder $^{\mathrm{a}}$ Amit Ram $^{\mathrm{a}}$ Yael Hershkovitz ${ }^{\mathrm{a}}$ David Tanne $^{\mathrm{b}, \mathrm{c}}$ \\ Natan M. Bornstein ${ }^{d}$ Ronen R. Leker ${ }^{e}$ Anat Horev ${ }^{f}$ Hen Hallevi $^{g}, h$ \\ Shlomi Peretz ${ }^{i}$ David Orion ${ }^{h, j}$ Inbar Zucker ${ }^{a, h}$
}

asrael Center for Disease Control, Israel Ministry of Health, Ramat Gan, Israel; bambam Health Care Campus, Haifa,

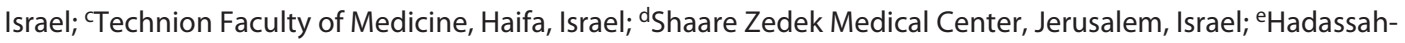

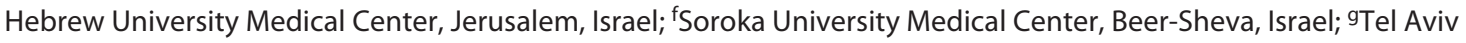

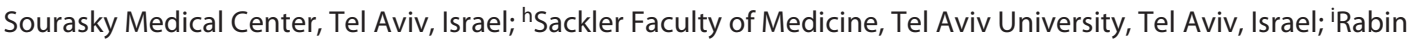

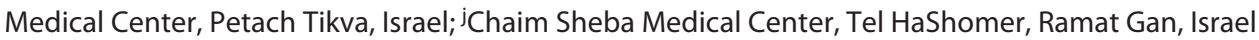

\section{Keywords}

Stroke $\cdot$ Stroke care $\cdot$ COVID-19 $\cdot$ Pandemic

\begin{abstract}
Introduction: The COVID-19 pandemic overwhelmed health-care systems worldwide, and medical care for other acute diseases was negatively impacted. We aimed to investigate the effect of the COVID-19 outbreak on admission rates and in-hospital care for acute stroke and transient ischemic attack (TIA) in Israel, shortly after the start of the pandemic. Methods: We conducted a retrospective observational study, based on data reported to the Israeli National Stroke Registry from 7 tertiary hospitals. All hospital admissions for acute stroke or TIA that occurred between January 1 and April 30, 2020 were included. Data were stratified into 2 periods according to the timing of COVID-19 restrictions as follows: (1) "pre-pandemic" - January 1 to March 7, 2020 and (2) "pandemic" - March 8 to April 30, 2020. We compared the weekly counts of hospitalizations between the 2 periods. We further investigated changes in demographic characteristics
\end{abstract}

and in some key parameters of stroke care, including the percentage of reperfusion therapies performed, time from hospital arrival to brain imaging and to thrombolysis, length of hospital stay, and in-hospital mortality. Results: 2,260 cases were included: 1,469 in the pre-COVID-19 period and 791 in the COVID-19 period. Hospital admissions significantly declined between the 2 periods, by $48 \%$ for TIA (rate ratio $[R R]=0.52 ; 95 \% \mathrm{Cl} 0.43-0.64)$ and by $29 \%$ for stroke (RR = $0.71 ; 95 \% \mathrm{Cl} 0.64-0.78)$. No significant changes were detected in demographic characteristics and in most parameters of stroke management. While the percentage of reperfusion therapies performed remained unchanged, the absolute number of patients treated with reperfusion therapies seemed to decrease. Higher in-hospital mortality was observed only for hemorrhagic stroke. Conclusion: The marked decrease in admissions for acute stroke and TIA, occurring at a time of a relatively low burden of COVID-19, is of great concern. Public awareness campaigns are needed as patients reluctant to seek urgent stroke care are deprived of lifesaving procedures and secondary prevention treatments.

(c) 2021 S. Karger AG, Base karger@karger.com

www.karger.com/ned

Karger ${ }^{\prime \prime}=$$$
\stackrel{2}{2}
$$

(c) 2021 S. Karger AG, Base
Correspondence to:

Carmit Libruder, carmit.libruder@moh.gov.il 


\section{Introduction}

On March 11, 2020, the novel coronavirus disease 2019 (COVID-19) was declared a pandemic by the World Health Organization [1]. The COVID-19 pandemic overwhelmed health-care systems worldwide, and medical care for other acute diseases was negatively impacted [2]. Containment measures, including social distancing and "stay home" campaigns, affected public use of health services. Stroke centers across the globe reported a reduction in the number of individuals with stroke symptoms attending the emergency departments, particularly those with transient ischemic attack (TIA) or mild symptoms [3].

In Israel, the first locally acquired case of COVID-19 was identified in the last week of February (epidemiological week 9), and by week 11, restrictions on the population included home quarantine to all persons returning from outside Israel and limitation of gatherings to a maximum of 100 people. Later on, restrictions were gradually extended, up to a nationwide lockdown that was issued on April 8 (epidemiological week 15). In regard to medical services, patients and health-care providers were encouraged to use telehealth services to reduce exposures. We aimed to investigate the impact of the COVID-19 outbreak on admission rates for acute stroke and TIA in Israel and to evaluate whether the management of acute stroke has been affected.

\section{Materials and Methods}

\section{Data Collection and Study Population}

We conducted a retrospective analysis based on data reported to the Israeli National Stroke Registry (INSR). The INSR contains data of all permanent residents of the State of Israel (age $\geq 18$ ) admitted to one of the general hospitals in the country and received a final diagnosis of acute stroke (ischemic and hemorrhagic) or TIA, according to the International Classification of Disease, ninth edition (ICD-9). Reporting to the INSR is mandatory. Regularly, on a quarterly basis, all hospitals provide the registry with an electronic report retrieved from their data systems, containing personalized clinical and administrative data on acute events of stroke/ TIA in patients hospitalized 6 months earlier. For the purpose of this study, earlier reporting to the INSR was required. We managed to get the collaboration of 7 tertiary hospitals that cover about $50 \%$ of stroke admissions in Israel. All 7 are among the 9 thrombectomy-capable centers in the country.

All hospital admissions due to acute stroke or TIA that occurred between January 1 and April 30, 2020 were included in the study. Acute events that occurred during hospital stay and cases that were transferred to one of the participating hospitals from another hospital were excluded.

In order to compare the period that was affected by the pandemic to the period that preceded it, data were stratified into 2 periods, according to the timing of COVID-19-related restrictions in Israel. The first period (pre-pandemic period) included patients admitted between January 1 and March 7, 2020. The second period (pandemic period) included patients admitted between March 8 and April 30, 2020. Missing data on some important variables including symptom onset times, tissue plasminogen activator administration times, and stroke severity, as measured by National Institutes of Health Stroke Scale (NIHSS) scores, were manually completed from hospital records or discharge letters, if available.

\section{Study Outcomes}

The primary outcome of our study was the weekly counts of hospital admissions, for acute stroke and TIA. Epidemiological weeks were defined as 7 days, beginning with Sunday and ending with Saturday, according to the CDC (MMWR) week numbering system. For this analysis, 16 weeks were included, for which we had full-week data. The weekly counts of hospitalizations for epidemiological weeks 2-10 (starting on January 5) were compared with those of epidemiological weeks 11-17 (starting on March 8). Secondary outcomes included some key parameters of stroke care as follows: the percentage of reperfusion therapies performed, time from the stroke onset to hospital arrival, time from hospital arrival to brain imaging, time from hospital arrival to thrombolysis, stroke severity (NIHSS scores), the percentage of patients admitted to a neurology ward, hospital length of stay, and in-hospital mortality.

\section{Descriptive Statistics}

Continuous variables were reported as medians with interquartile range or as means with standard deviation and were compared with $t$-tests or the appropriate nonparametric tests. Categorical variables were reported as proportions and were compared with Pearson's $\chi^{2}$ tests.

To graphically visualize the weekly counts of hospitalizations, we summarized the number of hospitalizations per week, for acute stroke and TIA separately, and a local polynomial regression smoothing function (locally estimated scatterplot smoothing) was fitted through the weekly numbers, using the loess function in $\mathrm{R}$ with default settings. The weekly numbers were plotted along with their approximate SEs, under the assumption that the numbers follow a Poisson distribution.

\section{Regression Analysis}

A Poisson regression model was used to estimate the effect of the COVID-19 pandemic on weekly counts of hospital admissions. Two separate regression models were fitted, one for stroke cases and another for TIA cases. In order to estimate whether the effect of the COVID-19 pandemic on hospitalization rates was greater than what might be expected on the basis of regular annual or seasonal trends, we adjusted for these variables. To represent the underlying annual trend, we added to the analysis hospitalization data for the years 2016-2019 (data retrieved from the INSR, with the same inclusion and exclusion criteria as for the 2020 analysis). The independent variable "year" was entered into the model as a continuous variable. To take seasonality into account, each year was divided to the same time periods as the year 2020: epidemiological weeks 2-10 represented period 1 and epidemiological weeks $11-17$ represented period 2 . The variable "period" was entered into the model as a categorical variable. We further defined a dummy variable to indicate the impact of the COVID-19 pandemic. The second period on 2020 was coded " 1 ." All previous periods, not affected by the pandemic, were coded " 0 ." The regression coefficient of this dummy variable provides an estimate of 
the ratio of the weekly hospitalization rate during the COVID-19 period to the weekly hospitalization rate in the pre-pandemic period, adjusted for the other predictors in the model [4]. In case of overdispersion of data, we used a negative binomial model.

The R statistical program (version 3.5.2) was used for the local polynomial regression, and all other statistical analyses were produced with SAS (version 7.1). A $p$ value of $<0.05$ was considered statistically significant.

\section{Ethics}

The INSR was authorized to collect personal clinical data for regulatory purposes, by means of a binding directive from the $\mathrm{Di}$ vision of Medicine in the Ministry of Health (MOH). The Helsinki Committee of the Israeli $\mathrm{MOH}$ approved the usage of anonymized clinical data, for research purposes, waiving the requirement for patient's informed consent.

\section{Results}

Between January 1 and April 30, 2020, 2,516 cases of cerebrovascular events were reported to the INSR. Eightynine events that occurred during hospital stay and 167 cases that were transferred from another hospital were excluded, leaving 2,260 cases eligible for the current study. Of them, 1,469 admissions occurred during the pre-pandemic period, and 791 admissions occurred during the pandemic period.

The weekly counts of hospital admissions for acute stroke and TIA, plotted along with their approximate SEs, are shown in Figure 1. Comparing between the 2 periods in 2020, the mean admission rate for acute stroke decreased from 116 to 82 cases per week, and the mean admission rate for TIA decreased from 37.9 to 19.9 cases per week. The number of weekly hospital admissions decreased by $29 \%$ for stroke and by $48 \%$ for TIA, as reflected in the unadjusted rate ratios (RRs) of 0.71 (95\% CI $0.64-0.78 ; p<0.001)$ and 0.52 (95\% CI $0.43-0.64 ; p<$ $0.001)$, respectively. Adjusting for annual and seasonal trends, in a model including hospitalization data of 4 previous years, there was a decrease of $34 \%$ in stroke hospitalizations $(\mathrm{RR}=0.66 ; 95 \%$ CI $0.59-0.73 ; p<0.001)$ and a decrease of $51 \%$ in TIA hospitalizations $(\mathrm{RR}=0.49 ; 95 \%$ CI $0.41-0.60 ; p<0.001)$ during the COVID-19 period. No seasonal effect was seen, neither for stroke admissions nor for TIA admissions $(p>0.10)$. A small but statistically significant annual trend was seen only for stroke admissions $(\mathrm{RR}=1.03$; 95\% CI 1.01-1.05; $p<0.001)$.

The reduction in hospital admissions during the COVID-19 period remained significant for both ischemic and hemorrhagic strokes $(\mathrm{RR}=0.74 ; 95 \%$ CI $0.66-0.82$ and $\mathrm{RR}=0.56$; $95 \% \mathrm{CI} 0.42-0.74$, respectively). The re-

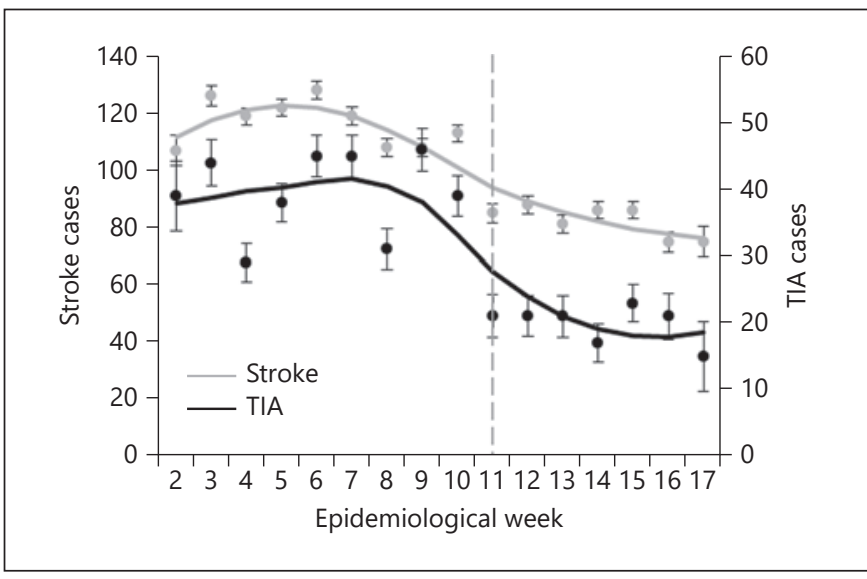

Fig. 1. Weekly hospital admissions for acute stroke and TIA in the pre-pandemic period (weeks $2-10$ ) versus the pandemic period (weeks 11-17). Left axis: the number of weekly stroke admissions (gray dots). Right axis: the number of weekly TIA admissions (black dots). A local polynomial regression smoothing function is fitted through the weekly numbers, with data points and SEs plotted. The vertical broken line on week 11 represents the start of the pandemic period. TIA, transient ischemic attack.

duction in cerebrovascular admissions was evident in all hospitals and reached statistical significance in 5 out of 7 centers (data not shown).

Demographic characteristics, including age and gender, were similar over the 2 periods in 2020 . The proportion of cases arriving by ambulance was significantly higher during the pandemic period (65.6\%) than during the pre-pandemic period (60.4\%). Diabetes was the single comorbidity that was significantly more common among patients hospitalized in the pre-pandemic period than among those hospitalized in the pandemic period (36.9 and $30.8 \%$, respectively). Stroke severity did not differ between the 2 periods (Table 1 ).

Among the ischemic stroke cases, no significant delay was noticed in the median time from the symptom onset to hospital arrival, between the 2 periods. Moreover, the proportion of patients arriving within $4.5 \mathrm{~h}$ from symptom onset (in time for thrombolysis) and within $24 \mathrm{~h}$ (in time for thrombectomy) was similar in both periods. Regarding the in-hospital management of ischemic stroke cases, no significant changes were detected in the time from hospital arrival to the first imaging nor to thrombolysis between the 2 periods. The percentage of reperfusion therapies performed also remained unchanged, while the median number of patients treated with reperfusion therapies decreased (from 20 to 15 cases per week; $p=0.35$ ).

A significant reduction in the length of hospital stay was detected during the pandemic period, compared with 
Table 1. Characteristics of patients admitted in the pre-pandemic period versus the pandemic period

\begin{tabular}{|c|c|c|c|}
\hline Variable & $\begin{array}{l}\text { Pre-pandemic } \\
(N=1,469)\end{array}$ & $\begin{array}{l}\text { Pandemic } \\
(N=791)\end{array}$ & $p$ value \\
\hline \multicolumn{4}{|l|}{ Demographics } \\
\hline Age, median years (IQR) & $74.2(65.2-83.0)$ & $73.4(65.1-82.3)$ & 0.28 \\
\hline Gender, males, $n(\%)$ & $807(54.9)$ & $445(56.3)$ & 0.55 \\
\hline Population group (Jews/Arabs), Jews, $n(\%)$ & $1,330(90.5)$ & $708(89.5)$ & 0.43 \\
\hline \multicolumn{4}{|l|}{ Stroke diagnosis, $n(\%)$} \\
\hline Ischemic stroke & $948(64.5)$ & $550(69.5)$ & 0.04 \\
\hline Hemorrhagic stroke & $169(11.5)$ & $72(9.1)$ & \\
\hline TIA & $352(24.0)$ & $169(21.4)$ & \\
\hline \multicolumn{4}{|l|}{ Comorbidities, $n(\%)$} \\
\hline Diabetes & $542(36.9)$ & $244(30.8)$ & 0.004 \\
\hline Hypertension & $896(61.0)$ & $451(57.0)$ & 0.07 \\
\hline Ischemic heart disease & $295(20.1)$ & $144(18.2)$ & 0.28 \\
\hline Hyperlipidemia & $637(43.4)$ & $332(42.0)$ & 0.52 \\
\hline Atrial fibrillation & $248(16.9)$ & $142(18.0)$ & 0.52 \\
\hline Arrival by ambulance, $n(\%)$ & $887(60.4)$ & $519(65.6)$ & 0.01 \\
\hline \multicolumn{4}{|l|}{ Stroke severity (NIHSS score)* } \\
\hline Median (IQR) & $4.0(2-9)$ & $4.0(2-8)$ & 0.94 \\
\hline$\leq 5, n(\%)$ & $389(41.0)$ & $234(42.5)$ & 0.81 \\
\hline $6-10, n(\%)$ & $113(11.9)$ & $64(11.6)$ & \\
\hline $11-15, n(\%)$ & $47(5.0)$ & $35(6.4)$ & \\
\hline $16-20, n(\%)$ & $53(5.6)$ & $27(4.9)$ & \\
\hline$>20, n(\%)$ & $27(2.8)$ & $15(2.7)$ & \\
\hline Missing, $n(\%)$ & $319(33.6)$ & $175(31.8)$ & \\
\hline
\end{tabular}

NIHSS, National Institutes of Health Stroke Scale; IQR, interquartile range; TIA, transient ischemic attack. * NIHSS scores were available only for ischemic strokes.

the pre-pandemic period. The median length of hospital stay decreased from 5 to 4 days among patients admitted with ischemic stroke and from 7 to 3 days among patients admitted with hemorrhagic stroke. The extent of the decline in the length of hospital stay for hemorrhagic strokes was reduced but remained statistically significant, after deduction of in-hospital deaths (data not shown). The percentage of patients admitted to a neurology ward was significantly higher in the COVID-19 period (68.3\%) than in the pre-COVID-19 period (54.5\%). Significantly higher in-hospital mortality rates were noticed for hemorrhagic strokes during the COVID-19 period, than during the pre-COVID-19 period (50.0 and $30.2 \%$, respectively). There was no apparent change in in-hospital mortality for ischemic strokes (Table 2).

\section{Discussion}

We demonstrated a significant reduction of $~ 34$ and $\sim 51 \%$ in hospital admissions for acute stroke and TIA, respectively, during the COVID-19 outbreak, which was evident across the country. Of note, this marked reduction occurred at a time of a relatively low COVID-19 burden, peaking at $\sim 700$ new COVID-19 cases per day and rapidly declining [5]. The steeper drop in hospital admissions for TIA implies that patients with less severe symptoms were less likely to seek medical care in a time of social isolation and fear of exposure to COVID-19. However, data suggest that patients with more significant strokes also avoided hospital visit as most characteristics of stroke patients hospitalized during the pandemic period, including stroke severity, were similar compared with those hospitalized in the pre-pandemic period.

As was found in our study, a reduction in the number of admissions for acute stroke and TIA during the pandemic period was evident in other countries [6-9]. Several studies reported no significant change in stroke severity during the pandemic [10-13], while others reported higher NIHSS scores during the pandemic period [7, 14] or a decline in admissions that was more evident among milder strokes $[6,15]$.

We found a reduced proportion of diabetics admitted in the pandemic period. This might be explained by a 
Table 2. Parameters of stroke-care and in-hospital management for patients admitted in the pre-pandemic period versus the pandemic period

\begin{tabular}{|c|c|c|c|}
\hline Variable & $\begin{array}{l}\text { Pre-pandemic } \\
(N=1,469)\end{array}$ & $\begin{array}{l}\text { Pandemic } \\
(N=791)\end{array}$ & $p$ value \\
\hline Admission to a neurology ward, $n(\%)$ & $801(54.5)$ & $540(68.3)$ & $<0.001$ \\
\hline \multicolumn{4}{|l|}{ Length of hospital stay, days } \\
\hline Hemorrhagic stroke & $n=169$ & $n=72$ & \\
\hline Median (IQR) & $7.0(3.0-13.0)$ & $3.0(1.0-8.0)$ & $<0.001$ \\
\hline Mean $\pm S D$ & $11.2 \pm 13.7$ & $6.7 \pm 11.7$ & $<0.02$ \\
\hline Ischemic stroke & $n=948$ & $n=550$ & \\
\hline Median (IQR) & $5.0(2.0-9.0)$ & $4.0(2.0-7.0)$ & $<0.001$ \\
\hline Mean \pm SD & $8.0 \pm 9.8$ & $6.2 \pm 7.5$ & $<0.001$ \\
\hline TIA & $n=352$ & $n=169$ & \\
\hline Median (IQR) & $2.0(1.0-4.0)$ & $2.0(1.0-3.0)$ & 0.24 \\
\hline Mean \pm SD & $3.2 \pm 4.5$ & $2.7 \pm 3.1$ & 0.11 \\
\hline \multicolumn{4}{|l|}{ In-hospital mortality, $n(\%)$} \\
\hline Hemorrhagic stroke & $51(30.2)$ & $36(50.0)$ & 0.003 \\
\hline Ischemic stroke & $82(8.6)$ & $41(7.5)$ & 0.42 \\
\hline \multicolumn{4}{|l|}{ Time frames* } \\
\hline \multicolumn{4}{|l|}{ Symptom onset to hospital arrival } \\
\hline Median hours (IQR) & $4.1(1.4-12.5)$ & $4.7(1.7-15.0)$ & 0.14 \\
\hline Arrived in $4.5 \mathrm{~h}, n(\%)$ & $305(32.2)$ & $178(32.4)$ & 0.98 \\
\hline Arrived in $24 \mathrm{~h}, n(\%)$ & $694(73.2)$ & $404(73.5)$ & 0.98 \\
\hline \multicolumn{4}{|l|}{ Hospital arrival to first imaging } \\
\hline Median minutes (IQR) & $65.0(28.0-164.0)$ & $63.0(28.0-137.0)$ & 0.35 \\
\hline \multicolumn{4}{|l|}{ Hospital arrival to thrombolysis } \\
\hline Median minutes (IQR) & $62.5(41.0-95.5)$ & $61.0(42.0-94.0)$ & 0.99 \\
\hline \multicolumn{4}{|l|}{ Reperfusion therapy* } \\
\hline Thrombolysis, $n(\%)$ & $121(12.8)$ & $79(14.4)$ & 0.38 \\
\hline Thrombectomy, $n(\%)$ & $97(10.2)$ & $59(10.7)$ & 0.76 \\
\hline Any reperfusion therapy, $n(\%)$ & $190(20.0)$ & $124(22.5)$ & 0.25 \\
\hline \multicolumn{4}{|l|}{ Any reperfusion therapy per week } \\
\hline Median (IQR) & $20(18-22)$ & $15(11-21)$ & 0.34 \\
\hline
\end{tabular}

IQR, interquartile range; TIA, transient ischemic attack; SD, standard deviation. * Relevant only for ischemic strokes.

greater fear of contagion among these patients, due to publications regarding an increased risk for severe COVID-19 disease among individuals with underlying medical conditions [16], and in particular diabetics [17, 18].

Similar to several other studies $[9,11,13,19]$, we found no significant change in the percentage of reperfusion therapies performed during the pandemic period, suggesting that in-hospital treatment was not negatively affected. However, the absolute number of reperfusion therapies performed seemed to decrease, as a result of the reduction in strokes admissions. The decline we observed in the median number of reperfusion therapies performed per week (from 20 to 15 treatments) did not reach statistical significance. Yet, as the reduction in ischemic stroke admissions was significant across stroke severities, we believe that the observed decrease is real, but we lacked the power to detect a statistically significant change.

On a national level, this raises a concern as possibly dozens of patients missed the opportunity for acute therapy with proven efficacy in reducing stroke-related death and disability. A significant decline in the number of reperfusion therapies performed during the pandemic was found in a large-scale research conducted in China [20], in a stroke network located in southern Europe [21], and in a Spanish multicenter study [12], with no reduction in the percentage of reperfusion therapies performed in any of these studies. Several smaller studies failed to detect significant reductions in the number of reperfusion therapies performed, although a trend toward decline was noticed $[6,22,23]$. 
The concern regarding the missed opportunity for treatment is not limited to severe strokes as even patients with milder strokes may benefit from acute stroke therapies and early assessment [24]. Furthermore, stroke and TIA patients reluctant to seek medical care most likely did not receive the appropriate secondary prevention treatments such as anticoagulation for atrial fibrillation, antiplatelet therapies, or blood pressure management [20]. Individuals who lost the opportunity of appropriate secondary prevention are at a higher risk of a recurrent event, with more severe outcomes $[3,20]$. Community healthcare providers should therefore put efforts for detecting those undiagnosed cases, and campaigns for increasing public awareness of stroke symptoms and the importance of timely treatment should be launched.

Our finding of a significantly higher in-hospital mortality rate for hemorrhagic strokes is not consistent with findings from other studies, possibly as most studies focused on ischemic stroke alone $[9,11,14,25]$, while others only provided the total stroke mortality rate $[7,23]$. An assessment of stroke severity was not documented for most hemorrhagic strokes; thus, whether the excess mortality is explained by higher proportion of severe cases needs to be further explored.

The fact that we did not observe significant changes in most parameters of in-hospital care, including time from hospital arrival to the first imaging and to thrombolysis, is reasonable as the spread of the COVID-19 in Israel during the examined period was not high. Although some of the internal wards were converted into coronavirus departments, hospitals were far from being overloaded and acute treatments were not impaired.

It is important to state that ever since July 2020 until January 2021, there has been a dramatic increase in the level of COVID-19 morbidity in Israel. This would probably affect in-hospital management of acute diseases like stroke, in a different manner than was observed in the current analysis. A follow-up research is therefore of high importance to examine the upcoming changes in the stroke admission rate and treatment management.

\section{Strengths and Limitations}

The main strength of our study is that it was carried within the framework of the INSR, a comprehensive national registry that covers all hospitalizations for acute cerebrovascular events in the country [26]. Due to time and logistic constraints, only 7 stroke centers were included in the study. However, these centers are spread over the country and cover about half of all stroke admissions in Israel, thus providing a broad dataset of national stroke incidence. We cannot rule out the possibility that some shifting of cases from one of the participating hospitals to another nonparticipating hospital occurred, but it is unlikely to explain a large portion of the decrease as informal reports from smaller centers also described a decline in hospital arrivals.

The main limitation of our study is inherent to its retrospective observational nature. Our results might not be generalized to other countries with a different COVID-19 burden or different response measures to the pandemic. Moreover, our study is limited to a relatively short followup period.

\section{Conclusion}

We demonstrated a marked reduction in hospital admissions for acute stroke and TIA that was evident shortly after the spread of the pandemic in Israel, despite a relatively low burden of COVID-19 in the country. Measures should be taken to minimize collateral damage caused by COVID-19 as patients reluctant to seek urgent stroke care are deprived of lifesaving procedures and secondary prevention treatments. Long-term follow-up is needed in order to evaluate possible changes in stroke outcomes, including recurrent stroke rates and strokerelated disabilities and mortality, in the era of COVID-19.

\section{Statement of Ethics}

Statement of ethics is provided in detail in the Materials and Methods section of the manuscript.

\section{Conflict of Interest Statement}

The authors have no conflicts of interest to declare.

\section{Funding Sources}

The authors did not receive any funding.

\section{Author Contributions}

All the authors contributed to the study, conception, and design. Material preparation, data collection, and analysis were performed by A.R., Y.H., C.L., and I.Z. The first draft of the manuscript was written by C.L. and I.Z. All the authors revised and commented on the previous versions of the manuscript. All the authors read and approved the final manuscript. All the authors are accountable for all aspects of the work. 


\section{References}

1 WHO. Virtual Press Conference on COVID-19; 2020 Mar 11 (Accessed 2020 August 30).

2 Zhao J, Rudd A, Liu R. Challenges and potential solutions of stroke care during the coronavirus disease 2019 (COVID-19) outbreak Stroke. 2020 May;51(5):1356-7.

3 Smith EE, Mountain A, Hill MD, Wein TH, Blacquiere D, Casaubon LK, et al. Canadian stroke best practice guidance during the $\mathrm{CO}$ VID-19 pandemic. Can J Neurol Sci. 2020;47: 474-8

4 Kuhn L, Davidson LL, Durkin MS. Use of Poisson regression and time series analysis for detecting changes over time in rates of child injury following a prevention program. Am J Epidemiol. 1994;140(10):943-55.

5 Glatman-Freedman A, Bromberg M, Ram A, Lutski M, Bassal R, Michailevich O, et al. A COVID-19 call center for healthcare providers: dealing with rapidly evolving health policy guidelines. Isr J Health Policy Res. 2020 Dec 2;9(1):73.

6 Diegoli H, Magalhães PSC, Martins SCO, Moro CHC, França PHC, Safanelli J, et al. Decrease in hospital admissions for transient ischemic attack, mild, and moderate stroke during the COVID-19 era. Stroke. 2020;51: 2315.

7 Kristoffersen ES, Jahr SH, Thommessen B, Rønning OM. Effect of COVID-19 pandemic on stroke admission rates in a Norwegian population. Acta Neurol Scand. 2020;142(6) 632-6.

8 Sharma M, Lioutas V-A, Madsen T, Clark J, O'Sullivan J, Elkind MS, et al. Decline in stroke alerts and hospitalisations during the COVID-19 pandemic. Stroke Vascular Neurol. 2020;5(4):403-5.

9 Tejada Meza H, Lambea Gil Á, Saldaña AS, Martínez-Zabaleta M, Juez PR, Martínez EL, et al. Impact of COVID-19 outbreak on ischemic stroke admissions and in-hospital mortality in North-West Spain. Int J Stroke. 2020 15(7):755-62
10 Rinkel LA, Prick JCM, Slot RER, Sombroek NMA, Burggraaff J, Groot AE, et al. Impact of the COVID-19 outbreak on acute stroke care. J Neurol. 2021;268(2):403-8.

11 Siegler JE, Heslin ME, Thau L, Smith A, Jovin TG. Falling stroke rates during COVID-19 pandemic at a Comprehensive Stroke Center: cover title: falling stroke rates during COVID-19. J Stroke Cerebrovasc Dis. 2020 May 14;29(8):104953.

12 Tejada Meza H, Lambea Gil Á, Saldaña AS, Martínez-Zabaleta M, Garmendia Lopetegui E, López-Cancio Martínez E, et al. Impact of COVID-19 outbreak in reperfusion therapies of acute ischaemic stroke in northwest Spain. Eur J Neurol. 2020;27(12):2491-8.

13 Teo KC, Leung WCY, Wong YK, Liu RKC, Chan AHY, Choi OMY, et al. Delays in stroke onset to hospital arrival time during COVID-19. Stroke. 2020 Jul;51(7):2228-31.

14 Agarwal S, Scher E, Rossan-Raghunath N, Marolia D, Butnar M, Torres J, et al. Acute stroke care in a New York City comprehensive stroke center during the COVID-19 pandemic. J Stroke Cerebrovasc Dis. 2020;29(9): 105068.

15 Perry R, Banaras A, Werring DJ, Simister R. What has caused the fall in stroke admissions during the COVID-19 pandemic? J Neurol. 2020;267:3457-8.

16 Yang J, Zheng Y, Gou X, Pu K, Chen Z, Guo $\mathrm{Q}$, et al. Prevalence of comorbidities and its effects in patients infected with SARS-CoV-2: a systematic review and meta-analysis. Int J Infect Dis. 2020 May;94:91-5.

17 Barron E, Bakhai C, Kar P, Weaver A, Bradley $\mathrm{D}$, Ismail $\mathrm{H}$, et al. Associations of type 1 and type 2 diabetes with COVID-19-related mortality in England: a whole-population study. Lancet Diabetes Endocrinol. 2020;8(10):81322.
18 Guo W, Li M, Dong Y, Zhou H, Zhang Z, Tian $\mathrm{C}$, et al. Diabetes is a risk factor for the progression and prognosis of COVID-19. Diabetes Metab Res Rev. 2020:e3319.

19 Esenwa C, Parides MK, Labovitz DL. The effect of COVID-19 on stroke hospitalizations in New York City. J Stroke Cerebrovasc Dis. 2020;29(10):105114.

20 Zhao J, Li H, Kung D, Fisher M, Shen Y, Liu R. Impact of the COVID-19 Epidemic on Stroke Care and Potential Solutions. Stroke. 2020;51:1996.

21 Montaner J, Barragán-Prieto A, Pérez-Sánchez S, Escudero-Martínez I, Moniche F, Sánchez-Miura JA, et al. Break in the stroke chain of survival due to COVID-19. Stroke. 2020; 51:2307.

22 Desai SM, Guyette FX, Martin-Gill C, Jadhav AP. Collateral damage: impact of a pandemic on stroke emergency services. J Stroke Cerebrovasc Dis. 2020;29(8):104988.

23 Rudilosso S, Laredo C, Vera V, Vargas M, Renú A, Llull L, et al. Acute stroke care is at risk in the era of COVID-19: experience at a comprehensive stroke center in Barcelona. Stroke. 2020;120:030329.

24 Markus HS, Brainin M. COVID-19 and stroke: a global World Stroke Organization perspective. Int J Stroke. 2020;15(4):361-4.

25 Kerleroux B, Fabacher T, Bricout N, Moïse M, Testud B, Vingadassalom S, et al. Mechanical thrombectomy for acute ischemic stroke amid the COVID-19 outbreak: decreased activity, and increased care delays. Stroke. 2020; 51(7):2012-7.

26 Ram A, Libruder C, Hershkovitz Y, Tanne D, Shohat T, Zucker I. [The Israeli national stroke registry: a tool for monitoring morbidity and acute stroke care in Israel]. Harefuah. 2019 Jun;158:352-6. 